\title{
Don d'organes: à vous d'améliorer la situation
}

II manque 1268 organes en Suisse. Soucieuse d'améliorer cette situation, Swisstransplant, la Fondation nationale suisse pour le don et la transplantation d'organes, a lancé mi-septembre une campagne nationale pour le don d'organes. Quelles en sont les raisons et pourquoi Swisstransplant mène-t-elle campagne dans les hôpitaux et dans les milieux qui gravitent autour de ceux-ci?

Franz Immer

Médecin spécialiste en chirurgie cardiaque et directeur de Swisstransplant
Correspondance: PD Dr Franz F. Immer Swisstransplant Laupenstrasse 37 Postfach 7952 CH-3001 Berne Tél. 0313808133 Fax 0313808132
Les médias grand public ont relaté dernièrement qu'il existe en Suisse un besoin urgent d'organes de donneurs. Ils ont ainsi ravivé une controverse dont les retombées sont clairement visibles dans les commentaires des versions en ligne des articles correspondants. Nous les avons lus pour prendre la température et identifier les malentendus. Vous serez de plus en plus confronté à ces arguments dans les prochains jours et les prochaines semaines, étant donné que nous avons commencé mi-septembre notre campagne afin de sensibiliser la population sur le thème du don d'organes et de l'urgence du besoin.

\section{En fait, je suis mort...}

Il faut expliquer la campagne et son concept avant de traiter les idées reçues les plus fréquentes à propos de la médecine de transplantation. Pour la première fois, nous lançons une campagne nationale: dans les espace publiques, affiches et présence dans les hôpitaux, les cabinets des médecins de famille et lors des congrès, distribution de documents informatifs et relations avec médias. Ainsi, nous empruntons volontairement une approche très directe: on voit des photos de personnes dans la fleur de l'âge; rien n'évoque la maladie et la mort. Au-dessus des photos se trouvent des titres grands, tels que «Je suis mort depuis 3 ans...» ou «Je suis mort depuis 5 mois...». On peut lire dans le texte supplémentaire: «... C'est vrai. Mais quelqu'un était là pour me faire don de son organe, ce qui me permet d'être encore en vie.»

\section{Où disparaît la volonté?}

Une raison explique pourquoi nous souhaitons nous faire passer notre message dans les milieux des hôpitaux et des cabinets médicaux: c'est là que l'on décide si la volonté du donneur se traduira par un don. L'année dernière, 37 organes au total ont été importés en Suisse alors que seulement sept organes de Suisse ont pu être attribués à un receveur étranger. Cette tendance existe depuis des années et continue de s'aggraver: si 504 patients ont encore été transplantés en 2011 (y compris donneurs vivants), le nombre des patients transplantés en 2012 ne s'élevait plus qu'à 453 $(-10 \%)$ et, sans les organes importés, à $416(-17 \%)$. On pourrait en conclure que la population suisse profite certes de la volonté de donneurs dans d'autres pays, mais qu'elle-même est trop peu disposée au don, ce qui dénoterait un manque de solidarité, pour ne pas dire de l'égoïsme. Or, ce n'est pas le cas. Notre population n'est généralement pas moins disposée au don que la population des pays voisins; la disposition au don d'organes est au contraire élevée dans toutes les régions linguistiques. Pourtant, on estime qu'environ 300 donneurs potentiels décèdent chaque année dans les unités de soins intensifs en Suisse. Ce nombre est plus de trois fois plus élevé que le nombre de donneurs signalés par les hôpitaux (en 2012, il s'agissait de 89 donneurs en état de mort cérébrale). Même si les intensivistes en Suisse pensent donc plus souvent au don d'organes que la moyenne européenne, de nombreux donneurs potentiels se perdent toutefois dans le processus de don. La cause principale est de toute évidence un taux de refus important, s'élevant à 52\% (avec des variations dans les grands hôpitaux entre $23 \%$ et $89 \%$ ), mais souvent également un manque de moyens dans les hôpitaux.

\section{De nombreux donneurs potentiels se perdent toutefois dans le processus de don.}

\section{Arguments contre les idées reçues}

Mener un entretien sur un possible prélèvement d'organes est une tâche difficile qui exige beaucoup de tact, notamment lorsque la décision ne peut plus être prise par la personne concernée elle-même. Nombreux sont les Suisses qui n'ont toujours pas de carte de donneur d'organes, confrontant ainsi leurs proches, déjà plongés dans un immense chagrin, au défi de prendre une décision qui, selon eux, correspond à la volonté de la personne décédée, et ce dans l'urgence. On comprend que cette situation n'est pas idéale pour prendre des décisions rationnelles et il y a là un risque de perdre des organes malgré la volonté du donneur. Plus vous vous familiarisez, en tant que 


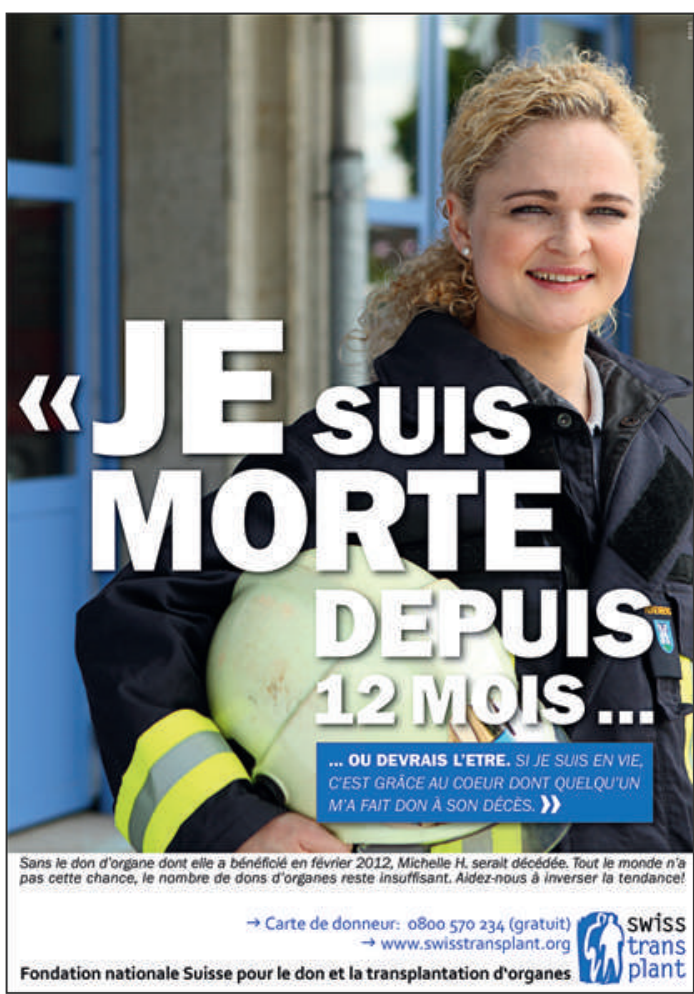

Pour sa campagne, Swisstransplant a choisi un approche très direct.

médecin, avec les objections et aux sensibilités à ce sujet ainsi qu'aux faits qui y sont liés, plus vous réussirez à rendre la discussion objective. Les objections les plus fréquentes sont les suivantes:

\section{Objection 1: Une mort cérébrale ne signifie pas être mort}

Les personnes n'appartenant pas au milieu médical confondent souvent mort cérébrale et coma. Lorsqu'elles lisent dans le journal des histoires de personnes qui se sont réveillées après des années de coma, elles en concluent que celles-ci auraient été assassinées si leurs organes avaient été prélevés. A l'inverse du coma, la mort cérébrale est un état irréversible, et les prélèvements d'organes sont uniquement réalisés en cas de mort cérébrale. Une personne est en état de mort cérébrale lorsque son cerveau n'est plus irrigué et qu'il s'arrête de fonctionner de manière irréversible. De l'extérieur, une personne en état de mort cérébrale ne se distingue pas d'une personne inconsciente: sa peau est rose, sa cage thoracique se soulève et s'abaisse, son cœur bat. Le système cardiovasculaire se maintient toutefois uniquement grâce à des appareils de soins intensifs. Si on arrête la respiration artificielle, le cœur et la circulation s'arrêtent également. Deux médecins doivent prouver, indépendamment l'un de l'autre, la mort cérébrale. Pour la diagnostiquer, ils effectuent une série d'examens standardisés. Conformément à l'or- donnance sur la transplantation d'organes, les examens cliniques stipulés dans les directives de l'ASSM doivent être utilisés, ceux-ci se fondant sur sept signes cliniques. On contrôle par exemple si des réflexes de base, commandés par le cerveau, tels que le réflexe de toux ou le réflexe de déglutition, fonctionnent encore. De plus, on vérifie que le patient ne recommence pas à respirer de lui-même lorsqu'on éteint le respirateur artificiel. La mort cérébrale est donc constatée avec certitude avant un prélèvement d'organes, et une mort cérébrale est une mort effective et irrémédiable, les organes ne pouvant être maintenus en vie qu'artificiellement.

\section{Objection 2: La transplantation d'organes est une prolongation des souffrances et non pas de la vie}

Les patients transplantés doivent prendre des immunosuppresseurs tout au long de leur vie, ces médicaments, comme tous les autres, ayant des effets indésirables. Il est erroné, ou plutôt prétentieux, de croire toutefois que ces effets indésirables sont si importants qu'une transplantation d'organes n'entraînerait en fait pas une prolongation de la vie, mais uniquement des souffrances. D'une part, notre expérience chez Swisstransplant montre que, chez la grande majorité des receveurs, une qualité de vie excellente est possible pendant des années. C'est d'ailleurs ce que nous souhaitons montrer avec les personnes au centre de notre campagne actuelle. D'autre part, considérer qu'il est mieux qu'une personne en souffrance meurt n'est défendable qu'au vu de sa propre vie et il est prétentieux ou du moins problématique de juger de la vie d'un autre. En tant que médecins, nous savons quelle volonté de vivre et quel amour de la vie animent un patient condamné s'il a une lueur d'espoir de pouvoir continuer à vivre grâce à un traitement. Seuls ceux qui l'ont vécu peuvent le comprendre: les personnes avec qui nous avons affaire tous les jours à Swisstransplant, parmi lesquelles 11 sont le visage de notre campagne. Il s'agit ici de rappeler qu'il est de notre devoir de tout faire pour permettre à nos patients de continuer à vivre. En d'autres termes, il convient de tout mettre en œuvre pour lutter contre les idées reçues afin de gagner un organe qui permet de prolonger la vie. En effet, comme tout autre domaine de la médecine, la médecine de transplantation continue également d'évoluer lorsqu'elle est appliquée, lors des transplantations. La qualité de vie des transplantés, nettement meilleure aujourd'hui qu'il y a encore quelques années, en est la preuve et des améliorations suivront encore.

\section{Objection 3: La transplantation d'organes est contraire à l'éthique}

Les réserves d'ordre éthique soulevées par la transplantation d'organes sont les plus difficiles à réfuter de manière rationnelle, car il s'agit ici d'une question fondamentale de l'existence humaine. L'«âme» d'une 
personne en état de mort cérébrale continue-t-elle à vivre? Est-il juste de se substituer au destin et de sauver la vie d'une personne en lui faisant profiter de la mort d'une autre personne? L'âme d'une personne peut-elle reposer en paix, si ses organes continuent de vivre dans une autre personne? Nous devons faire
Nous pouvons également assurer qu'on ne laisse pas mourir un blessé grave qui possède une carte de donneur dans le but de prendre ses organes. Le don d'organes est seulement possible en cas de mort cérébrale et si celle-ci a été confirmée par des médecins indépendants. De plus, les victimes d'accidents mortels

\section{Conformément à l'ordonnance sur la transplantation d'organes, les examens cliniques stipulés dans les directives de l'ASSM doivent être utilisés, ceux-ci se fondant sur sept signes cliniques.}

avec ces questions, nous disposons «uniquement» des faits médicaux et ceux-ci prouvent clairement la mort cérébrale comme un événement irréversible, comme la mort qui nous définit comme personne. A l'inverse, l'«âme» ne peut pas être mesurée, elle est différente pour chaque individu et chacun de ces concepts est légitime. Il serait faux et contre-productif de vouloir influencer l'avis de patients pour les «convaincre» du don d'organes. Par contre, il est de notre devoir d'informer ouvertement et clairement les proches d'une personne en état de mort cérébrale sur le don d'organes, notamment pour respecter la volonté de la personne décédée et pour soulager ses proches.

Parallèlement à ces préoccupations éthiques fondamentales, il faut également tenir compte de l'éthique en termes de justice: l'attribution des organes est-elle équitable, c'est-à-dire qu'une personne riche et influente n'a-t-elle pas plus de chance de bénéficier d'un don d'organes que le «citoyen lambda»? Le scandale en Allemagne a étayé ces soupçons. En Suisse, il est cependant clairement établi que les structures de Swisstransplant garantissent une répartition juste, reposant uniquement sur les critères des groupes d'experts en tenant compte de l'urgence, du bénéfice médical, du temps sur la liste d'attente et du besoin. sont souvent si gravement blessées qu'elles perdent tout leur sang et que la question du don d'organe ne se pose même pas. Le don d'organes n'entre en ligne de compte que si les traitements n'ont aucune chance de succès et que le cerveau a cessé de fonctionner entièrement et de manière irréversible.

J'espère avoir réussi à montrer que le don d'organe ne pose pas de problème sur le plan éthique. Dans le quotidien professionnel, il convient également de le garder présent à l'esprit, de recueillir et de respecter la volonté du donneur potentiel de manière claire et transparente aux services des urgences et unités de soins intensifs: il s'agit là d'une condition pour offrir aux personnes sur liste d'attente la chance d'une deuxième vie. Merci beaucoup pour le travail que vous accomplissez dans cet esprit!

\section{Articles interactifs}

Vous souhaitez commenter cet article? II vous suffit pour cela d'utiliser la fonction «Ajouter un commentaire» dans la version en ligne. Vous pouvez également consulter les remarques de vos confrères sous: www.bullmed.ch/ numero-actuel/articles-interactifs/ 